Cal cul at i on of nonl i near eddy-cur rent probl ens by the har moni $c$ bal ance finite el ement method

\begin{tabular}{|l|l|}
\hline 著者 & Yamada Sot oshi , Bi ri nger P. P. , Bessho Kazuo \\
\hline $\begin{array}{l}\text { j our nal or } \\
\text { publ i cat i on t i t l e }\end{array}$ & I EEE Tr ansact i ons on Magget i cs \\
\hline vol une & 27 \\
\hline number & 5 \\
\hline page $r$ ange & $4122-4125$ \\
\hline year & $1991-09-01$ \\
\hline URL & ht t p: //hdl . handl e. net /2297/48339 \\
\hline
\end{tabular}




\title{
CALCULATION OF NONLINEAR EDDY-CURRENT PROBLEMS BY THE HARMONIC BALANCE FINITE ELEMENT METHOD
}

\author{
Sotoshi YAMADA, † Paul P. BIRINGER and Kazuo BESSHO \\ Faculty of Technology, Kanazawa University, Kodatsuno 2-40-20, Kanazawa, Japan \\ $\dagger$ Dept. of Electrical Engineering, University of Toronto, Ontario, M5S 1A4, Canada
}

\begin{abstract}
The harmonic balance finite element method proposed by us solves the nonlinear time-periodic solution expressed as the sum of fundamental and harmonic components. This paper' describes a new procedure for calculation which emphasizes the field computation in the harmonic domain. As a result, the improvement of reducing memory and calculation time is achieved.
\end{abstract}

\section{INTRODUCTION}

We have proposed the harmonic balance finite element method (HBFEM) for the sleady-state analysis of nonlinear dynamic magretic fields in the harmonic domain (Irequency domain)[1]. Unlike the step-by-step method and the others [2],[3], the HBFEM solves time-periodic nonlinear dynamic field analysis without time-derivative calculation. The results in the harmonic domain obtained by the HBFEM are suited for the design of electromagnetic. devices. Because the frequency response is important in the dynamic characteristics of the system.

In the previous HBFEM, all harmonic components are șimultaneously computed. Therefore, the system matrix of the HBFEM expands in size when the number of harmonics to be considered increases.

It is well-known in physical systems that the amplitude of each harmonic component decreases with increasing order of the harmonics. By taking note of the property and introducing in iterative process, we can separate the system matrix of the HBFEM at each harmonic component. The procedure of the calculation makes the HBFEM more efficient and efiective.

\section{FORMULATION}

We consider nonlinear eddy-current problems in 2-dimensional Cartesian coordinates. And only time-periodic solution is assumed on condition that a magnetizing current varies periodically with time.

Using Galerkin's method, the magnetic vector potential $\mathrm{A}=$ $(0,0, A)$ in 2-D Cartesian coordinates satisfies the following integral:

$$
\begin{aligned}
& \left.\int\right\}\left\{\frac{\partial N_{1}}{\partial x}\left(\nu \frac{\partial A}{\partial x}\right]+\frac{\partial N_{J}}{\partial y}\left[\nu \frac{\partial A}{\partial y}\right]\right\} d x d y \\
& -\not \int N_{J}\left(J_{0}-0 \frac{\partial A}{\partial t}\right) d x d y=0
\end{aligned}
$$

where $\nu$ is the magnetic reluctivity depending on the magnelic field. $N_{\mathrm{i}}$ is the shape function of the first-order triangular element as a weighting function.

The Weierstrass Approximation Theory states that any periodic continuous variable can be approximated by the or thonormal function. We can express the time-periodic vector potential and other variables by the trigonometric function in this problems. Then, the vector potential and the magnetizing current density can be written:

$$
\begin{aligned}
A & =\sum\left\{A_{n} \sin (n \omega t)+A_{n c} \cos (n \omega t)\right\} \\
& =1,3,5 \ldots
\end{aligned}
$$

$$
\begin{aligned}
J_{0} & =\sum\left\{J_{n g} \sin (n \omega t)+J n c \cos (n \omega t)\right\} \\
n=1.3 .5 \ldots &
\end{aligned}
$$

In order to consider the saturation and hysteresis characteristics of the core, the magnetizing curve is expressed as

$$
H=H(B, \sigma B / d t)
$$

When the time-periodic flux density is given, we calculate the waveform of the magnetic reluctivity during a single cycle and obtain in the Fourier expansion

$$
\begin{aligned}
\nu(t) & =H[B(t)] / B(t) \\
& =\nu 1+\Sigma[\nu n \sin (n \omega t)+\nu n \theta \cos (n \omega t)] \\
& n-2,1,0, \ldots
\end{aligned}
$$

where $\nu_{0}, \nu_{n s}$, and $\nu_{n c}$ are the coefficients of the Fourier transformation.

The vector potential $A$ in one element surrounded with node points $i=1,2,3$ is

$$
A=A^{1} N_{1}+A^{2} N_{2}+A^{3} N_{3}
$$

$N_{i}$ is the shape function as

$$
\begin{gathered}
N i=\left(a_{1}+b_{1} x+c_{1} y_{1}\right) / 2 \Delta \\
\left(a_{1}=x_{1} y_{x}-x_{k} y_{1}, b_{1}-y_{j}-y_{k}, c_{1}=x_{k}-x_{j}\right)
\end{gathered}
$$

where $\left(x_{i}, y_{i}\right)$ is the displacement of the node $i$ and $\Delta$ is the cross section of an element.

For the weighting function $N_{j}(j=1,2,3)$, we calculate the first and second terms of Eq.(1), thus

$$
\begin{aligned}
& \text { Jf }\left\{\frac{\partial N_{1}}{\partial x}\left(\nu \frac{\partial A}{\partial x}\right)\right\} d x d y=\sum_{1-1,2,3} \frac{b_{1} b_{1}}{1 \Delta} * \\
& \text { * }\left(d_{1} A_{1} A^{\prime}+d_{12} A_{1} 0^{\prime}+d_{13} A_{33}{ }^{1}+d_{1} A_{3} A_{3} 0^{\prime}+d_{15} A_{5} 3^{\prime}+\cdots\right) \sin \omega t+ \\
& \left(d_{2} A_{1} A^{\prime}+d_{22} A_{1} 0^{\prime}+d_{23} A_{3}{ }^{\prime}+d_{2} A_{3} A_{3} c^{\prime}+d_{25} A_{5} 3^{\prime}+\cdots\right) \cos \omega t+ \\
& \left(d_{3} A_{1} g^{\prime}+d_{32} A_{1} c^{\prime}+d_{33} A_{3} 3^{3}+d_{3} A_{3} A^{\prime}+d_{35} A_{5} g^{\prime}+\cdots\right) \sin 3 \omega t+ \\
& \left(d_{1} A_{3} g^{\prime}+d_{42} A_{1} a^{\prime}+d_{43} A_{3} g^{\prime}+d_{4} A_{3} 0^{\prime}+d_{48} A_{5} 3^{\prime}+\cdots\right) \cos 3 \omega(+\cdots)
\end{aligned}
$$$$
\iint\left\{\frac{\partial N_{I}}{\partial y}\left(\nu \frac{\partial A}{\partial y}\right]\right\} d x d y=\sum_{t, 1,2,3} \frac{c, c_{i}}{t \Delta} *
$$

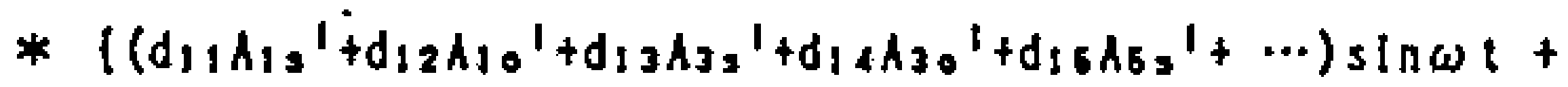$$
\left(d_{21} A_{1} a^{\prime}+d_{22} A_{1} 0^{l}+d_{23} A_{3} a^{l}+d_{2} A_{13} A_{3}{ }^{\prime}+d_{25} A_{5} a^{\prime}+\cdots\right) \cos \omega t+
$$$$
\left(d_{3} A_{1},{ }^{3}+d_{32} A_{30}{ }^{i}+d_{33} A_{3}{ }^{1}+d_{3} d_{1} A_{30}{ }^{i}+d_{35} A_{5}{ }^{1}+\cdots\right) \sin 3 \omega 1+
$$

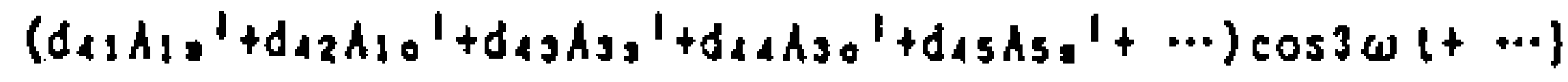

Finally, the third term with time-derivalive is derived by

$$
\begin{aligned}
& \int \mathrm{N}_{\mathrm{j}}\left(\mathrm{J}_{0}-\sigma \frac{\partial A}{\partial t}\right) \mathrm{dxdy} \\
& \left.\left.\left.=\left[(\Delta)_{13} / 3+\sigma \omega(8) 1 A_{1} 0^{1}+8\right] 2 \lambda_{1} 0^{2}+8\right]_{3} A_{10} 0^{3}\right) \Delta / 12\right] \sin \omega t+ \\
& \left.\left.\mid \Delta A_{1 c} / 3-\sigma \omega\left(8 ; 1 A_{1},{ }^{1}+g_{j} A_{1},{ }^{2}+g_{j} 3 A_{1},{ }^{3}\right) \Delta / 12\right\} \cos \omega\right\}+ \\
& \mid \Delta)_{3 j} / 3+3 \sigma \omega\left(8 j \mid A_{3} \mathrm{e}^{3}+8 i 2 A_{3} \mathrm{c}^{2}+8 j 3 A_{3} \mathrm{c}^{3}\right) \Delta / 12 \mid \sin 3 \omega l+ \\
& \left.\left.\left[\Delta J_{30} / 3-3 \sigma \omega\left(B_{1} A_{3} 3^{1}+8\right)_{2} A_{3} 3^{2}+B_{1} 3 A_{3} 3^{3}\right) \Delta / 12\right] \cos 3 \omega(+\cdots)\right]
\end{aligned}
$$


where $g_{i j}$ is given by

$$
8 i j=\left\{\begin{array}{lll}
2 & -11 & -i=j \\
1 & \text { if } & i \neq j
\end{array}\right.
$$

As the trigonometric function is one of the orthonormal functions, the coefficients of $\sin \omega t$ and $\cos \omega t$ are equated separately to zero. Moreover, we arrange the equations for $N_{j}(j=1,2,3)$ and the matrix equation is derived by

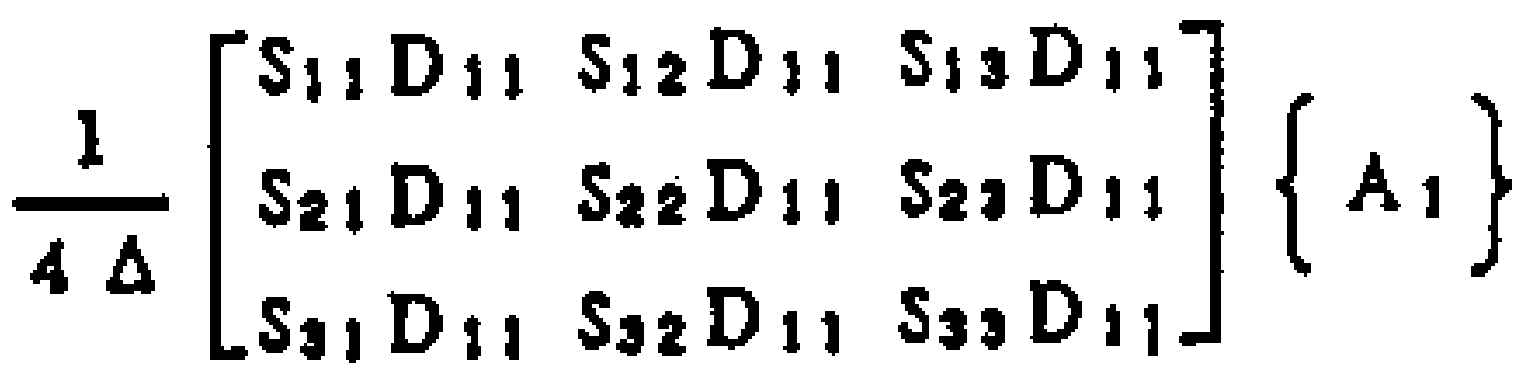

$$
\begin{aligned}
& +\frac{\sigma \omega \Delta}{12}\left[\begin{array}{ccc}
2 N_{1} & N_{1} & N_{1} \\
N_{1} & 2 N_{1} & N_{1} \\
N_{1} & N_{1} & 2 N_{1}
\end{array}\right]\left\{A_{1}\right\} \\
& =\underset{3=3.5,7 \ldots 4 \Delta}{-\Sigma_{1}} \frac{1}{4 \Delta}\left[\begin{array}{lll}
S_{11} D_{11} & S_{12} D_{11} & S_{13} D_{11} \\
S_{21} D_{11} & S_{22} D_{11} & S_{23} D_{11} \\
S_{32} D_{11} & S_{32} D_{13} & S_{33} D_{13}
\end{array}\right]\left\{A_{3}\right\}+\left\{K_{1}\right\}
\end{aligned}
$$

where the column vectors $\left\{A_{j}\right\},\left\{K_{1}\right\}$ and $s_{i j}$ are given by

$$
\begin{gathered}
\left.\left(A_{j}\right)=\mid A_{j 3}{ }^{2} A_{j c^{3}} A_{j a^{2}} A_{j c^{2}} A_{j s^{3}} A_{j c^{3}}\right\}^{\top}(10 a) \\
\left\{K_{1}\right)=\Delta / 3\left|J_{13} J_{10} J_{13} J_{10} J_{13} J_{10}\right|^{\top} \quad(10 b) \\
{\left[\begin{array}{llll}
s_{11} & s_{12} & s_{13} \\
s_{21} & s_{22} & s_{23} \\
s_{21} & s_{32} & s_{33}
\end{array}\right]=\left[\begin{array}{lll}
b_{1} b_{1}+c_{1} c_{1} & b_{1} b_{2}+c_{1} c_{2} & b_{1} b_{3}+c_{1} c_{3} \\
b_{2} b_{1}+c_{2} c_{1} & b_{2} b_{2}+c_{2} c_{2} & b_{2} b_{3}+c_{2} c_{3} \\
b_{3} b_{1}+c_{3} c_{1} & b_{3} b_{2}+c_{3} c_{2} & b_{3} b_{3}+c_{3} c_{3}
\end{array}\right]}
\end{gathered}
$$

The coefficients of $\sin (h \omega t)$ and $\cos (h \omega t)$ are also arranged in the same way and we have the matrix equation: where

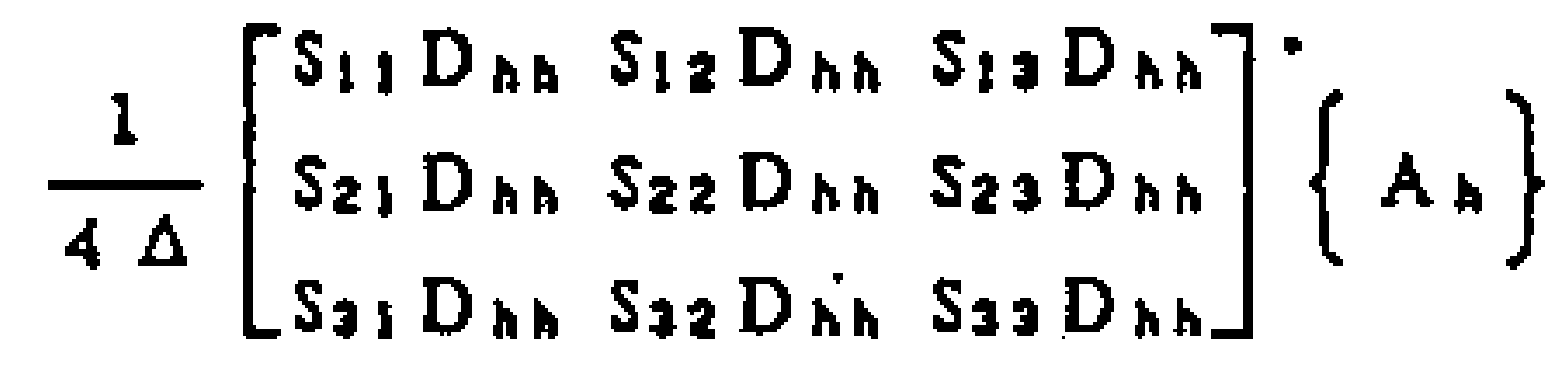

$$
\begin{aligned}
& +\frac{\sigma \omega \Delta}{12}\left[\begin{array}{ccc}
2 N_{h} & N_{h} & N_{h} \\
N_{h} & 2 N_{h} & N_{h} \\
N_{h} & N_{h} & 2 N_{h}
\end{array}\right]\left\{A_{s}\right\}
\end{aligned}
$$

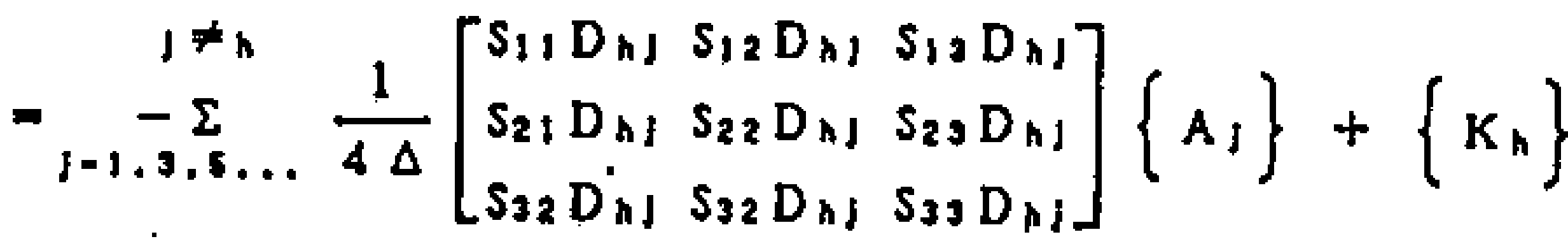

where

$$
\left(K_{h}\right)=\Delta / 3\left\{J_{h s} J_{h e} J_{h s} J_{h e} J_{h s} J_{h e}\right\}^{T}
$$

Here, the coefficient $d_{i j}$ in Eq.(7) and the block matrix $D_{h j}$ in Eqs.(9) and (11) are concerned with the magnetic reluctivity and are given by

$$
\begin{aligned}
D & =\left[\begin{array}{cccc}
D_{11} & D_{13} & D_{13} & \cdots \\
D_{31} & D_{33} & D_{35} & \cdots \\
D_{51} & D_{53} & D_{66} & \cdots \\
\vdots & \vdots & \vdots & \ddots
\end{array}\right] \\
& =\left[\begin{array}{ccccc}
d_{11} & d_{12} & d_{13} & d_{14} & \cdots \\
d_{21} & d_{22} & d_{23} & d_{21} & \cdots \\
\hdashline d_{31} & d_{32} & d_{33} & d_{34} & \cdots \\
d_{11} & d_{12} & d_{13} & d_{14} & \cdots \\
\vdots \vdots & \vdots & \vdots & \vdots & \ddots
\end{array}\right]
\end{aligned}
$$

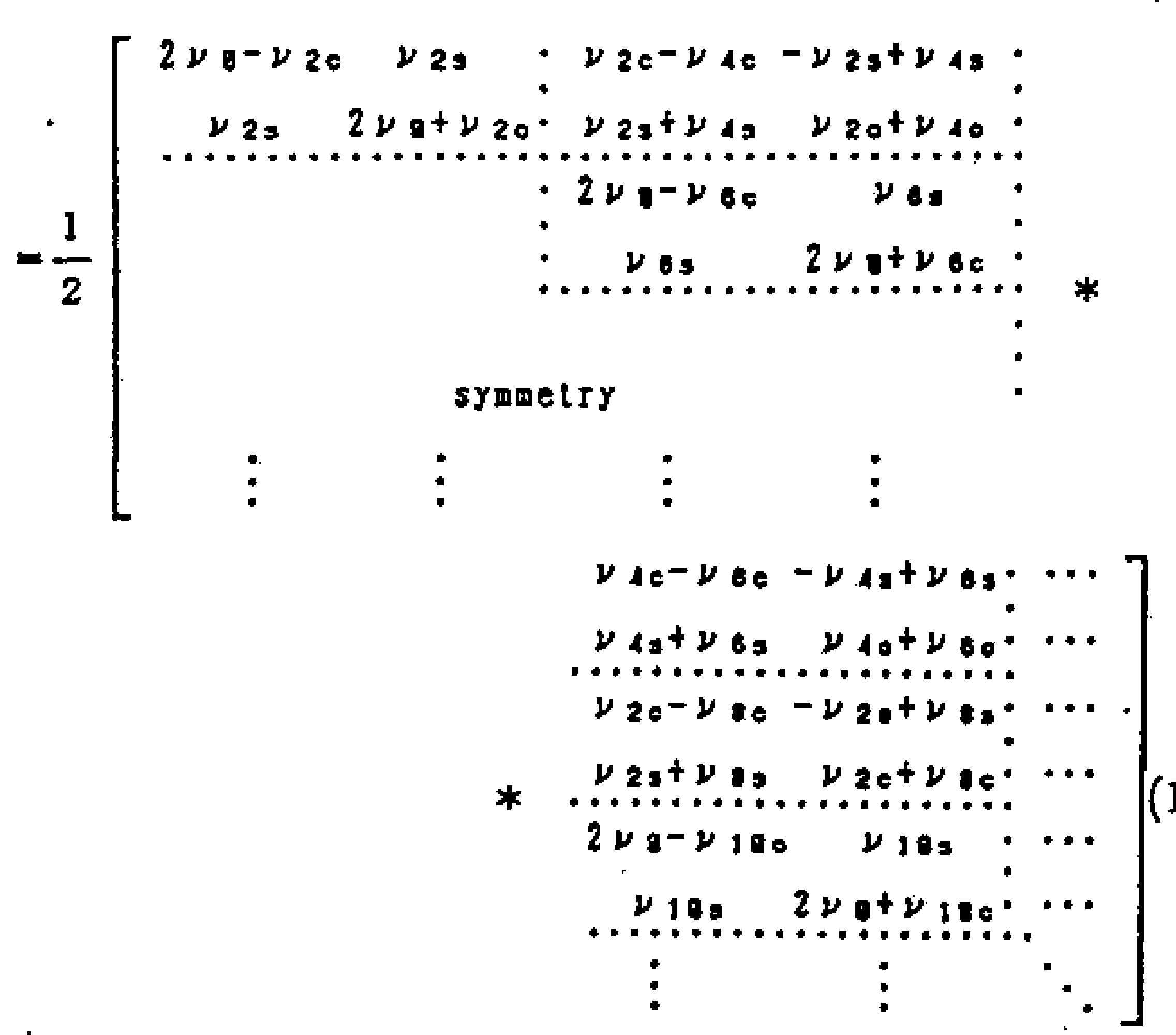

Moreover, the block matrix $N_{h}$ is

$$
N_{A}=\left[\begin{array}{cc}
0 & -h \\
h & 0
\end{array}\right]
$$

The system equation for the entire region of interest is arranged by the conventional FEM procedure. Based on Eq.(9), the system equation for the fundamental components is

$$
\left.\left[H_{11}\right]\left(A_{1}\right)^{*}=\sum_{1=3.6 \ldots}-\left[H_{1}\right]\right]\left(A_{1}\right\}^{k-1}+\left|G_{1}\right|
$$

For the h-th order harmonic components, we have in the same way

$$
\left.\left[H_{n A}\right]\left(A_{n}\right)^{k}=\sum_{j=1.3 \ldots}^{\mid N h}-\left[H_{n}\right]\right]\left(A_{j}\right\}^{x-1}+\left\{G_{n}\right\}
$$

We apply the iterative calculation process to Eqs.(15) and (16). The superscripts $k$ and $(k-1)$ denote the $k$ and $(k-1)$-th iterations. In order to solve Eq.(15) for the fundamental comporents, we assume the components for other harmonics based on the $(k-1)$-th solution. Then, the right-hand side term becomes known. With the identical operation, the higher components can be also obtained in turn. The process is continued until the con. vergence is satisfied.

It is the valid approximation in eddy-cutrent problems that higher components decrease rapidly with increasing the order of harmonic. Therefore, it is enough to continue to calculate harmonics up to finite order within an arbitrary margin of error.

\section{CALCULATION PROCEDURE AND DISCUSSES}

Figure 1 shows the flow chart in the calculation procedure of the HBFEM as mentioned above. To start with, the solution for the fundamental components is sought supposing that harmonic components have initial values. From the next step, the harmonics generated by the nonlinearity are calculated successively. The lower harmonic components are the dominant, hence we can neglect the calculation of the higher components by taking into account the margin of error.

Let us examine the size of the system matrix as shown in Eqs.(15) and (16). Note that these matrix equations are not solved simultaneously. Therefore, the required cache memory is only for a set of the system matrix. Assume that the freedom of 
4124

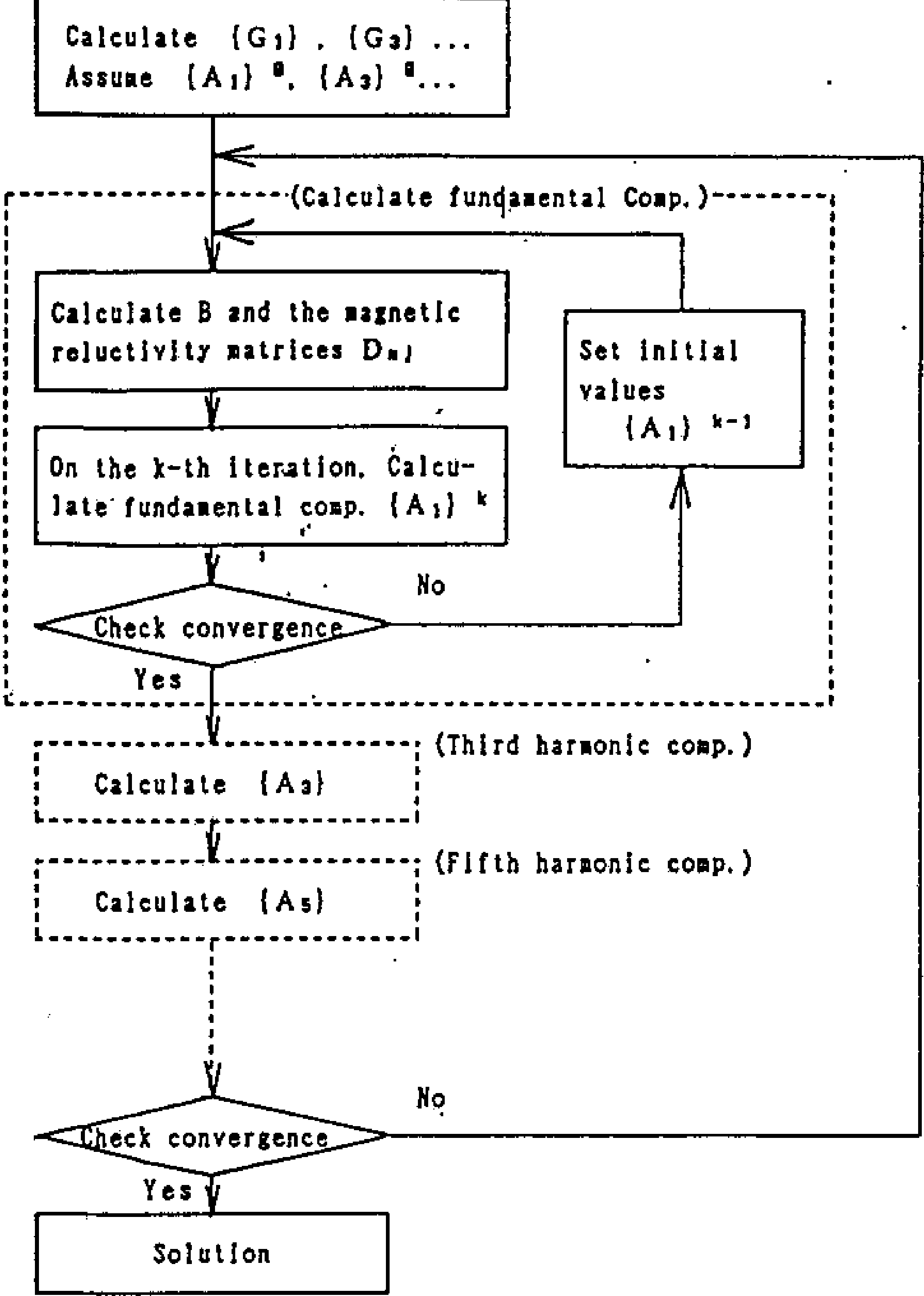

Fig. 1 Flow chart of HBFEM

a problem is $n$ (the number of unknown potentials) and harmonics up to $(2 m-1)$-th order are required. The earlier HBFEM procedure have the memory $M_{1}$ for the system matrix [1]

$$
M_{1}=4 k_{B} \mathrm{~nm}^{2} \text { (words) }
$$

where $k_{B}$ denotes the band with of the sparse matrix. On the other hand, the new approach requires a cache memory of $M_{2}$ :

$$
M_{2}=4 k_{B} n \text { (words) }
$$

By comparing the two cases, the proposed approach decreases the cache memory by the ratio $1 / \mathrm{m}^{2}$. When the order of the system equation decreases by the ratio $1 / m$, then the calculation time using the Gaussian elimination method is roundly reduced to $1 / \mathrm{m}^{3}$. The procedure of the HBFEM requires the $m$ times calculation of Eqs.(15) and (16). Thus, the time required at each iteration is approximately $1 / \mathrm{m}^{2}$ less than at the previous HBFEM. Note that the number of ilerations required to reach convergence is not considered here.

In the analysis of the HBFEM, we have the following merits:

1. We can examine the flux distribution at each harmonic.

2. It is easy to recognize the nonlinear effect.

3. The analyzed result is used for the frequency response which is suited for the system design.

4. When the nonlinearity is relatively week, this approach is much more memory-saving and timesaving.
IEEE TRANSACTIONS ON MAGNETICS, VOL. 27, NO. 5, SEPTEMBER 1991

\section{ANALYSIS OF ELECTROMAGNET WITH SHADING COIL}

We apply the new approach of the HBFEM to an electromagnet with shading coil as shown in Fig.2. The magnetic core is made of ferrite and the magnetizing characteristic is approximated in Fig.3. Eddy currents are induced in the shading coil and the region of the shading coil is analyzed as the eddy-current problem.

The HBFEM including the harmonics up to fifth order is applied to the calculation. The region for calculation is a half of the cross section in Fig.2. The numbers of node and triangular element are 506 and 921 respectively. The convergence condition is defined by

$$
\left|\frac{A_{h}(k)-A_{h}(k-1)}{A_{\text {nax }}(k)}\right|<0.005
$$

where $A_{\text {max }}^{(k)}$ denotes the maximum values of the vector potential in the iteration times $k$. The parameters for the calculation are given by

$$
\begin{aligned}
& \text { Hagnetizing current density: } \\
& \qquad \begin{aligned}
J_{15} & =1.00 \times 10^{8} \mathrm{~A} / \mathrm{m}^{2} \\
J_{35} & =-5.4 \times 10^{4} \mathrm{~A} / \mathrm{m}^{2} \\
J_{5} & =J_{10}=J_{30}=J_{50}=0.0 \\
\text { Conductivity of shading coll: } & \\
\sigma & =3.8 \times 10^{7} \mathrm{~s} / \mathrm{m}
\end{aligned}
\end{aligned}
$$

Frequency :
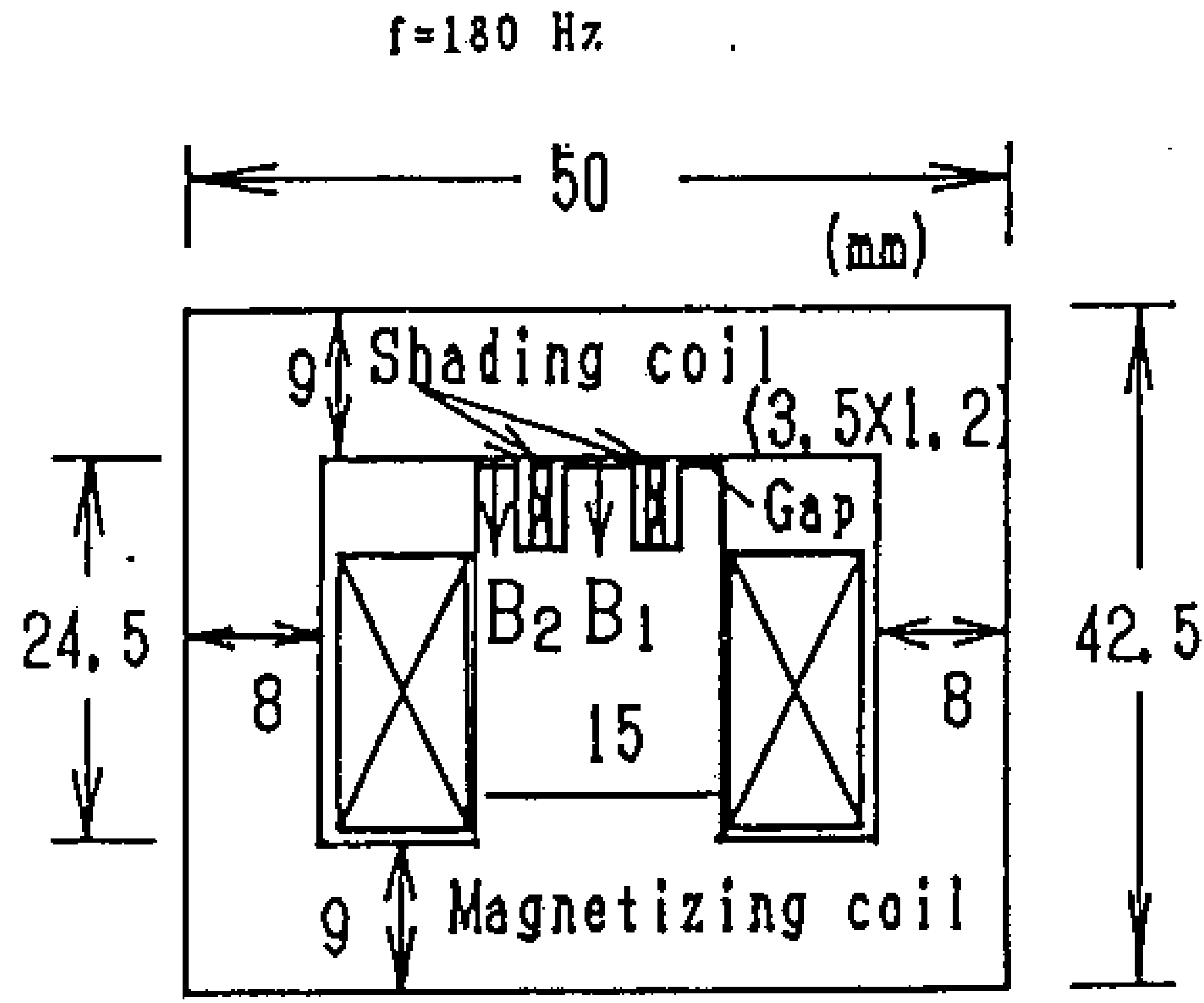

Gap length $=0,19$, Depth $=15$

Fig.2 Electromagnet with shading coil<smiles>[AlH2]</smiles>

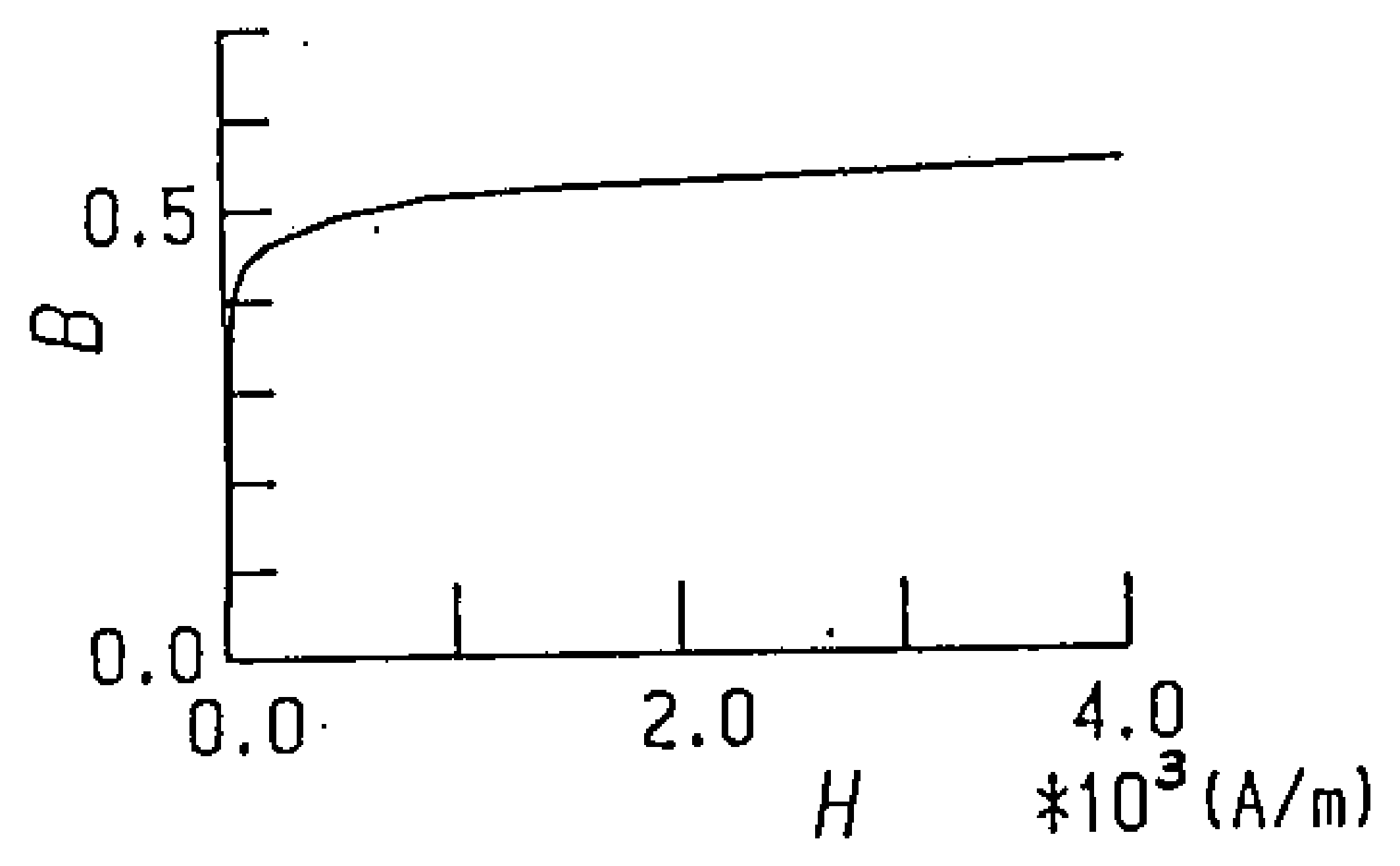

Fig. 3 Magnetizing curve (Ferrite) 


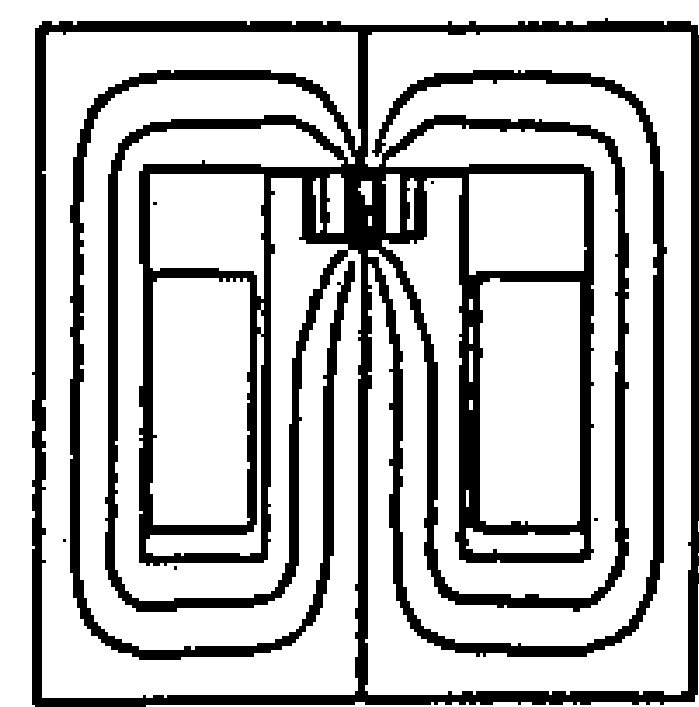

$\omega l=0$

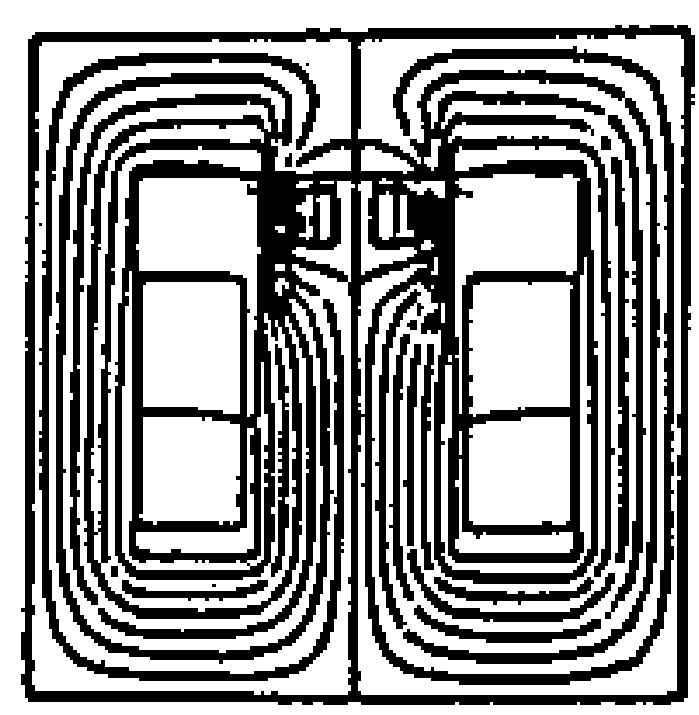

$\omega t=\pi / 1$

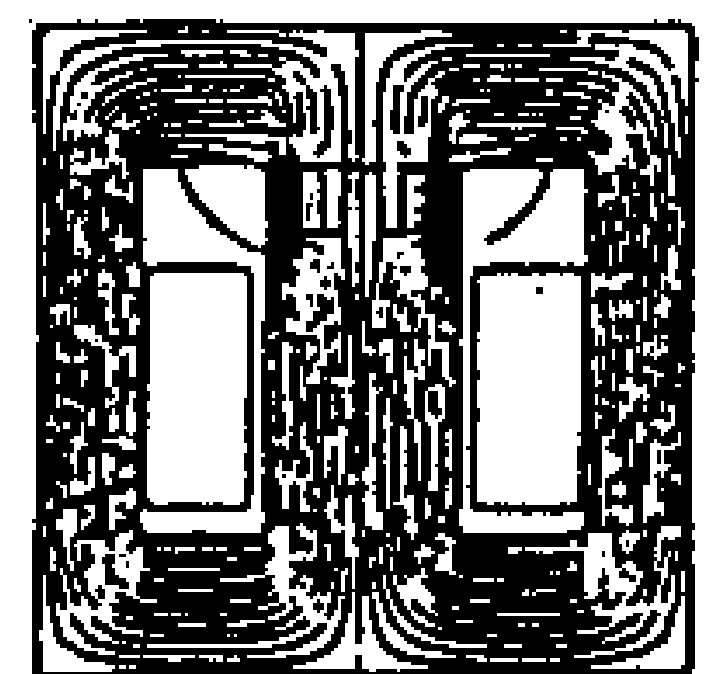

$\omega t=\pi / 2$

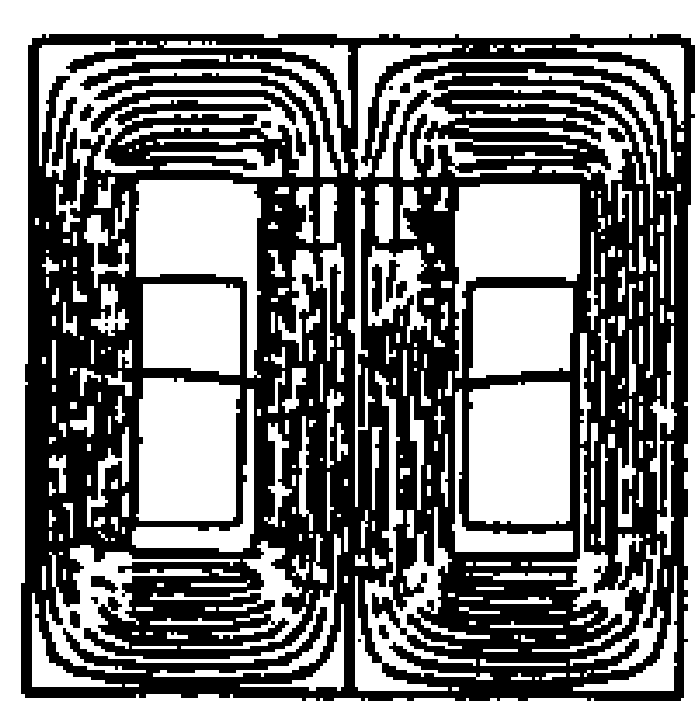

(a) Fundamental comp. $\omega t=3 \pi / 1$

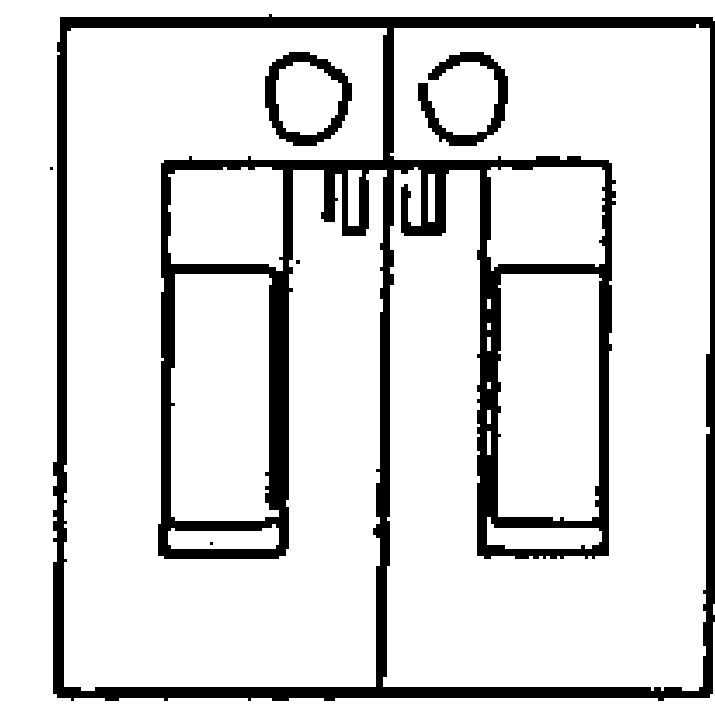

$3 \omega t=0$

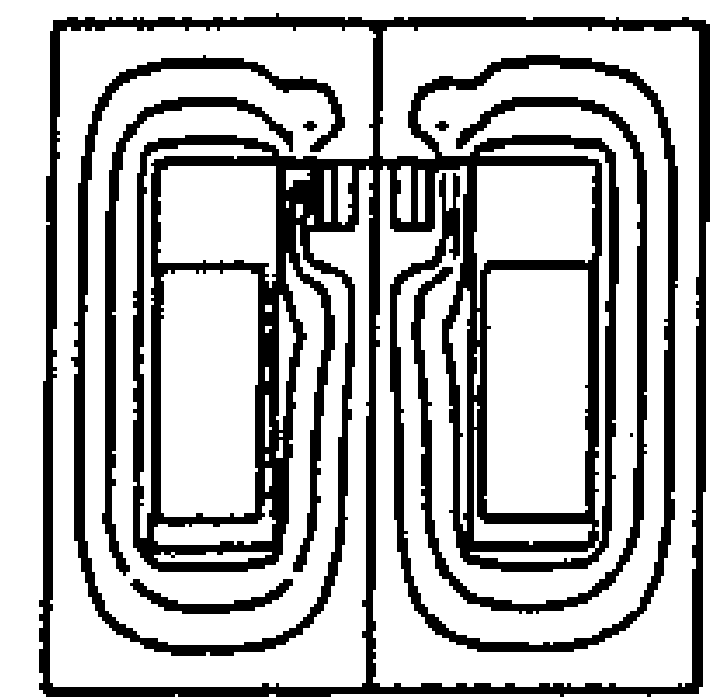

$3 \omega t=\pi / 1$

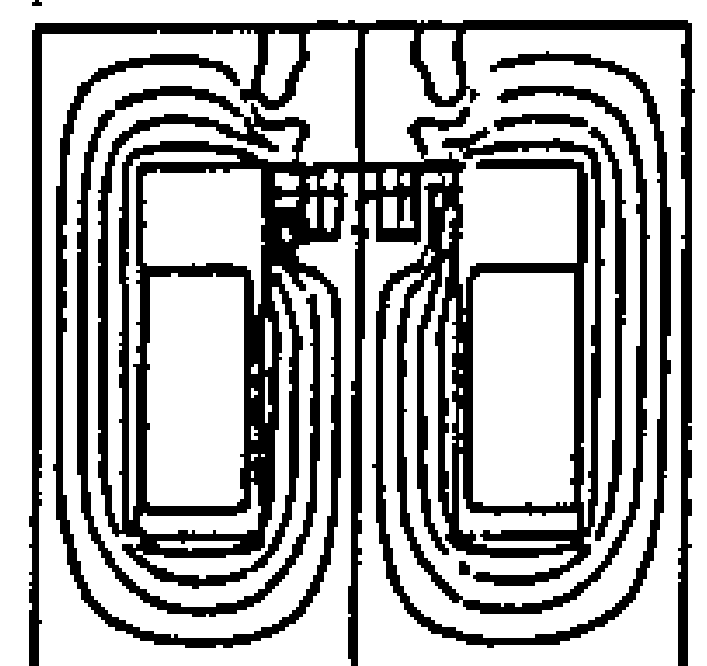

$s \omega t=\pi / 2$

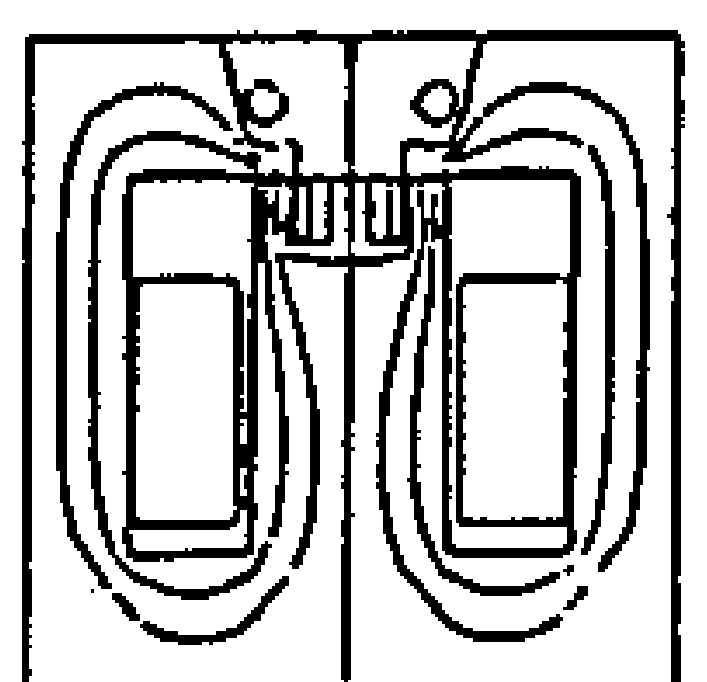

$3 \omega t=3 \pi / 1$

(b) Third comp.

Fig.4 Flux distribution .

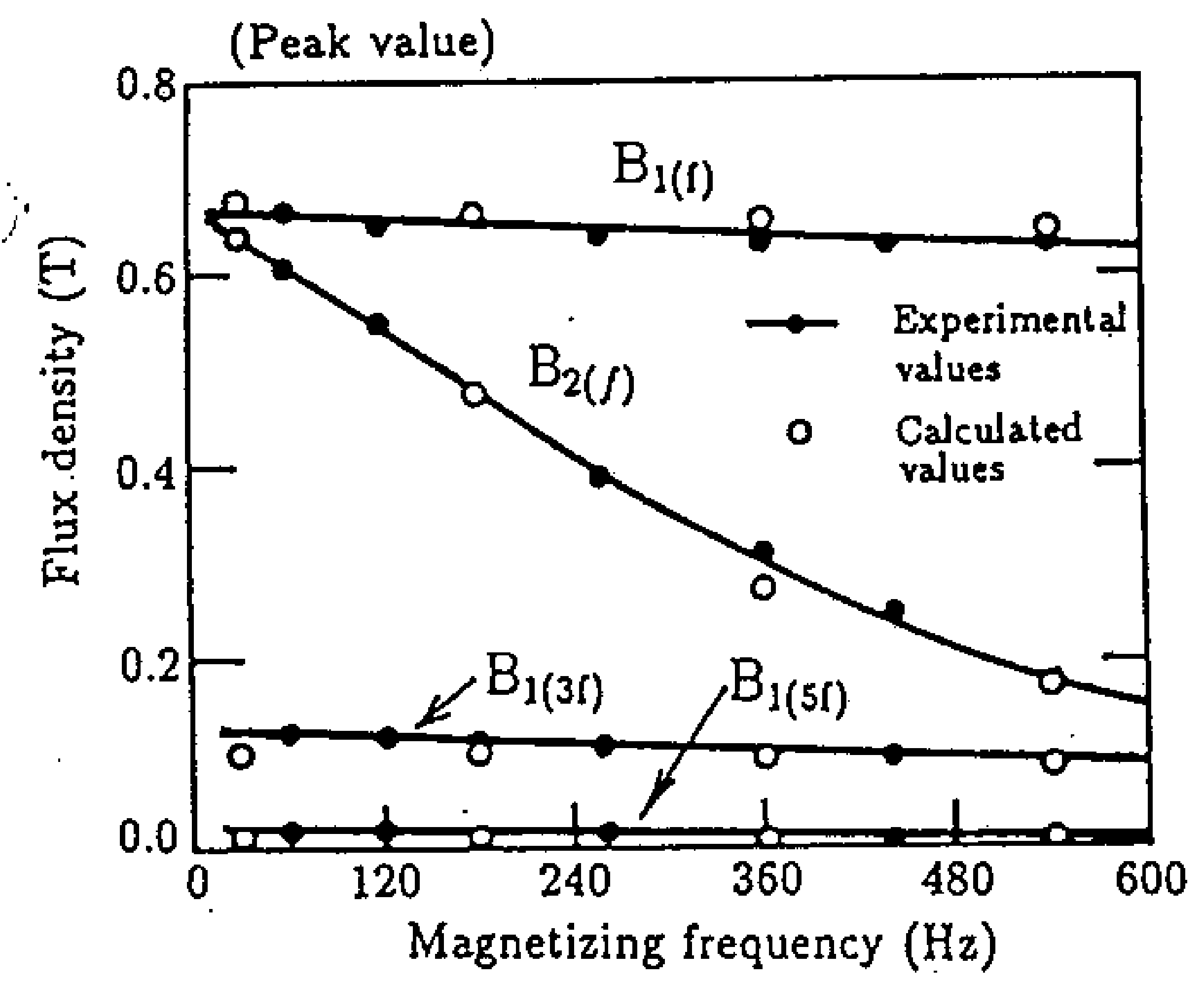

$B_{1}$ : Flux density outside shading coil

$B_{2}:$ Flux density inside shading coil

Fig. 5 Harmonic components of flux density
Table 1 Number of iterations

\begin{tabular}{c|cc}
\hline \multirow{2}{*}{$\begin{array}{c}\text { Erequency } \\
(\mathrm{Hz})\end{array}$} & \multicolumn{2}{|c}{ Numbér of } \\
\cline { 2 - 3 } & Previous HBFEM & Ner HBFEH \\
\hline 30 & 79 & 180 \\
180 & 74 & 112 \\
360 & 78 & 101 \\
540 & $76^{\circ}$ & 132 \\
\hline
\end{tabular}

The equi-potential lines of the fundamental, third and fifth harmonics in Fig.4 are drawn at the phase $h \omega t=0, \pi / 4, \pi / 2$ and $3 \pi / 4(h=1,3,5)$. The magnetic core outside the shading coil reaches the saturation level. Then, the harmonic component of the flux are generated there. The magnetic flux inside the shading coil is rejected and delayed because of the shading coil.

Next, as magnetizing frequency is changed from 30 to $540 \mathrm{~Hz}$, the harmonic components of a flux density are analyzed and compared with the experimental results. Figure 5 shows the harmonic components of the flux densities on both the calculated and experimental results. $B_{1}$ and $B_{2}$ indicate the flux densities outside and inside the shading coil. $B_{1}$ includes the harmonic components because of the saturation outside the shading coil. Meanwhile, $B_{2}$ has only the fundarnental component and decreases with increasing the frequency.

The new approach of the HBFEM makes efforts to decrease the cache memory for calculation. But we are uncertain of convergence in the iterative approach. Table 1 shows the comparison between the new approach and the previous HBFEM. The results imply that the number of iteration increases roughly. However, the reduction of the matrix in size overcomes the defect.

\section{CONCLUSIONS}

The calculation procedure for the HBFEM proposed here is to calculate each frequency componerit in the harmonic domain. The procedure reduces the memory requirement needed for the solution of the system matrix. As the nonlinearity is relatively weak, the procedure of the HBFEM becomes more effective and timesaving.

\section{REFERENCES}

[1] S.Yamada, K.Bessho, J.Lu, "Harmonic Balance Finite Element Method to Nonlinear AC Magnetic Analysis", IEEE Trans. Magnetics, Vol.MAG-25, No.4, pp95-99(1989).

[2] F.Bouillault, A.Razek, "Dynamic Model for Eddy Current" Calculation in Saturated Electric Machines", IEEE Trans. Magnetics, Vol.MAG-19, No.6, pp2639-2642(1983).

[3] T.Hara, T.Naito, J.Umoto, "Time-Periodic Finite Element Method for Nonlinear Diffusion Equation", IEEE Trans. Mag* netics, Vol.MAG-21, No.6, pp2261-2262(1985). 